Supporting Information

\title{
Atomic Structure of Self-Assembled Monolayer of Thiolates on a Tetragonal $\mathrm{Au}_{92}$ Nanocrystal
}

\author{
Chenjie Zeng, ${ }^{\dagger}$ Chong Liu, ${ }^{\ddagger}$ Yuxiang Chen,${ }^{\dagger}$ Nathaniel L. Rosi, ${ }^{\star}$ and Rongchao Jin ${ }^{*} \dagger$ \\ 'Department of Chemistry, Carnegie Mellon University, Pittsburgh, Pennsylvania 15213, US \\ ${ }^{*}$ Department of Chemistry, University of Pittsburgh, Pittsburgh, Pennsylvania 15260, US
}

\section{Experiments}

Chemicals. Tetrachloroauric(III) acid $\left(\mathrm{HAuCl}_{4} \cdot 3 \mathrm{H}_{2} \mathrm{O}, 99.99 \%\right.$ metals basis, Aldrich), 4-tertbutylbenzenethiol (TBBT, 97\%, Alfa Aesar), Sodium borohydride ( $\mathrm{NaBH}_{4}, 99.9 \%$, Aldrich). Tetrahydrofuran (THF, HPLC grade, 99.9\%, Aldrich), Methanol (HPLC grade, 99.9\%, Aldrich), $\mathrm{CH}_{2} \mathrm{Cl}_{2}$ (HPLC grade, 99.9\%, Aldrich), Toluene (HPLC grade, 99.9\%, Aldrich), Acetonitrile (HPLC grade, 99.9\%, Aldrich). All chemicals were used as received.

Synthesis of the $\mathrm{Au}_{92}(\text { TBBT })_{44}$ nanocrystal. In the first step, $\mathrm{HAuCl}_{4} \cdot 3 \mathrm{H}_{2} \mathrm{O}(0.11 \mathrm{mmol}, 45 \mathrm{mg})$ and TBBT thiol $(0.55 \mathrm{mmol}, 90 \mu \mathrm{L})$ were mixed in $10 \mathrm{~mL}$ methanol, and stirred for 30min to form yellowish precipitate of $\mathrm{Au}(\mathrm{I})$-TBBT complexes. The $\mathrm{Au}(\mathrm{I})$-TBBT complexes were collected and redispersed in 10 $\mathrm{mL}$ THF by sonication. The reaction mixture was stirred gently and cooled down to $-4{ }^{\circ} \mathrm{C}$. Then $\mathrm{NaBH}_{4}$ $\left(0.8 \mathrm{mmol}, 30 \mathrm{mg}\right.$ dissolved in $1 \mathrm{~mL}$ cold $\left.\mathrm{H}_{2} \mathrm{O}\right)$ was injected to the reaction mixture all at once. The color of the solution changed to black within $5 \mathrm{~s}$, indicating the reduction of $\mathrm{Au}(\mathrm{I})-\mathrm{TBBT}$ complexes into polydispersed $\mathrm{Au}_{x}(\mathrm{TBBT})_{y}$ nanoparticles. After reacting for 18 hours, the THF solvent was removed, and the $\mathrm{Au}_{x}(\mathrm{TBBT})_{y}$ nanoparticles were separated by first precipitating with methanol, and then extracting with $\mathrm{CH}_{2} \mathrm{Cl}_{2}$. In the second step, $\sim 25 \mathrm{mg}$ of $\mathrm{Au}_{x}(\mathrm{TBBT})_{y}$ nanoparticles were dissolved in $1 \mathrm{~mL}$ toluene and $100 \mu \mathrm{L}$ TBBT thiol, and stirred at $60^{\circ} \mathrm{C}$ for 24 hours. Then, additional $100 \mu \mathrm{L}$ of TBBT thiol was added and stirred at $80{ }^{\circ} \mathrm{C}$ for another $24 \mathrm{hr}$. After that, the nanoparticles were collected by first precipitating with methanol and then extracting with $\mathrm{CH}_{2} \mathrm{Cl}_{2}$. The $\mathrm{Au}_{92}(\mathrm{TBBT})_{44}$ nanoparticles were crystallized by vapor diffusion of $\mathrm{CH}_{3} \mathrm{CN}$ into a toluene solution of the nanoparticles. Rhombic black crystals were obtained and characterized by X-ray crystallography.

Characterization. Electrospray ionization mass spectra (ESI-MS) were collected on a Waters Q-TOF mass spectrometer equipped with Z-spray source. The source temperature was kept at $70{ }^{\circ} \mathrm{C}$. The sample was directly infused into the chamber at $5 \mu \mathrm{L} / \mathrm{min}$. The spray voltage was kept at $2.20 \mathrm{kV}$ and the cone voltage at $60 \mathrm{~V}$. The sample was dissolved in toluene and diluted $(1: 2 \mathrm{v})$ by dry methanol (containing 50 $\mathrm{mM} \mathrm{CsOAc}$ to form $\mathrm{Cs}^{+}$/cluster adducts in the ESI process). UV-Vis spectra were recorded on a HewlettPackard (HP) Agilent 8453 diode array spectrophotometer at room temperature.

$X$-ray Crystallography. Single crystal X-ray diffraction data of $\mathrm{Au}_{92}(\mathrm{TBBT})_{44}$ were collected on a Bruker X8 Prospector Ultra equipped with an Apex II CCD detector and an $\mathrm{I} \mu \mathrm{S}$ micro-focus $\mathrm{CuK}_{\alpha}$ X-ray source $(\lambda=1.54178 \AA)$. A piece of black plate-shaped crystal with dimensions $0.20 \times 0.12 \times 0.01 \mathrm{~mm}$ was mounted onto a MiTeGen MicroMeshes with fluorolube. The data were collected under cold $\mathrm{N}_{2}$ flow at $230 \mathrm{~K}$. A monoclinic unit cell with dimensions $\mathrm{a}=45.3000(11), \mathrm{b}=28.6539(7), \mathrm{c}=88.045(2), \alpha=\gamma=$ $90^{\circ}, \beta=90.940(2)^{\circ}$ was derived from the least-square refinement of 9580 reflections in the range of 2.701 $<\theta<51.202$. Centrosymmetric space group $\mathrm{C} 2 / \mathrm{c}$ was determined based on intensity statistics and systematic absences. Data were collected to $0.95 \AA$ and truncated to $0.99 \AA$ resolution due to weak diffraction. After integration of the data by Bruker program SAINT, empirical absorption correction was applied using program SADABS. Maximum and minimum transmittance (Tmax, Tmin) values are 0.6623 and 0.0403 , respectively. 
The structure was successfully solved by direct methods and refined with full-matrix least squares on $\mathrm{F}^{2}$ using SHELXTL software package. All the $\mathrm{Au}, \mathrm{S}$ atoms were located easily, the $\mathrm{C}$ atoms were generated via subsequent difference Fourier syntheses. Idealized atom positions were calculated for all hydrogen atoms (with d- $\left(\mathrm{C}_{\text {methyl }}-\mathrm{H}\right)=0.979 \AA$, d- $\left.\left(\mathrm{C}_{\text {phenyl }}-\mathrm{H}\right)=0.95 \AA\right)$. All the $\mathrm{Au}, \mathrm{S}$ atoms and phenyl $\mathrm{C}$ atoms were refined anisotropically. The tert-butyl $\mathrm{C}$ atoms and all $\mathrm{H}$ atoms were refined isotropically. Benzene rings were constrained to perfect hexagons using AFIX 66 command. The tert-butyl groups were refined as rigid bodies with the quaternary carbon as "pivot" atoms. ADP restraints were applied to phenyl $\mathrm{C}$ atoms using SIMU command. Rigid bond restraints were also applied to phenyl $\mathrm{C}$ atoms using DELU command. ISOR command was used to restrain C1, C91 with NPD problems. All the refinement parameters are summarized in the following tables.

\section{Supporting Figures and Tables}

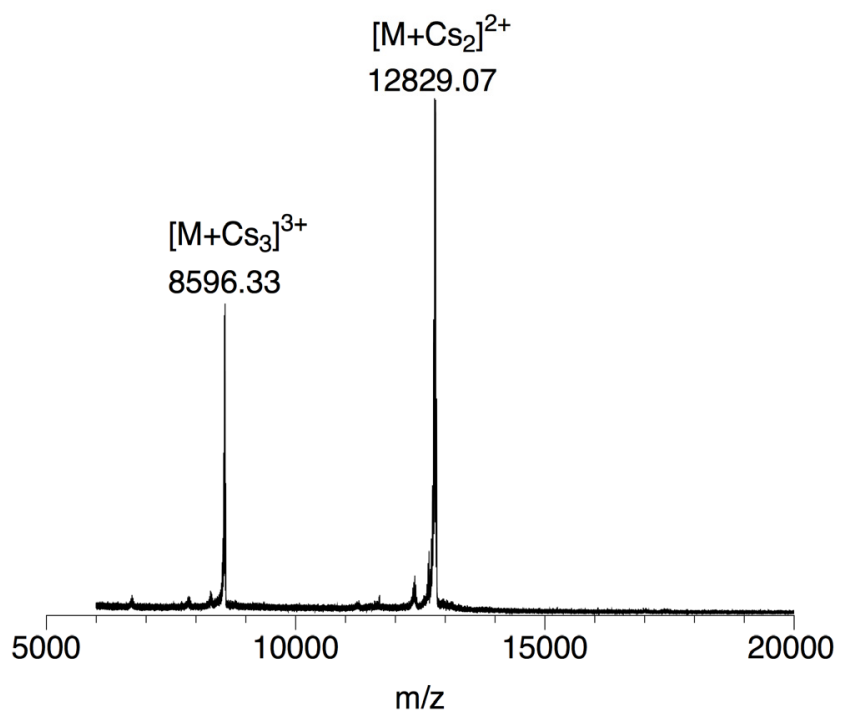

Figure S1. ESI-MS of the $\mathrm{Au}_{92}(\mathrm{TBBT})_{44}$ nanocrystal. The experimentally determined molecular weight of the nanocrystal is 25392.6 (based on the $3+$ peak) and 25389.99 (based on the $2+$ peak). The theoretical Mw of $\mathrm{Au}_{92}(\mathrm{TBBT})_{44}$ is 25392.79 .

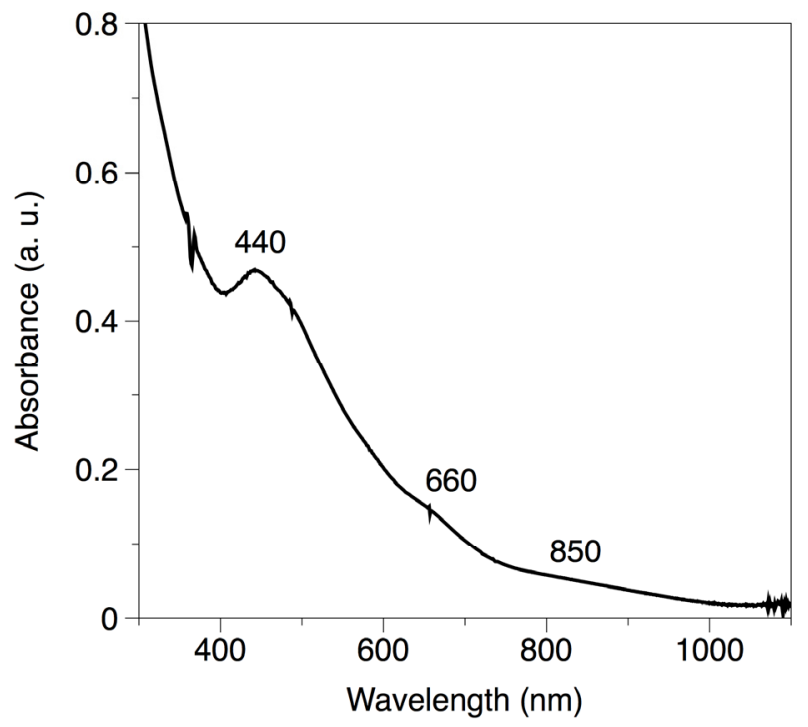

Figure S2. UV-vis absorption spectrum of $\mathrm{Au}_{92}(\mathrm{TBBT})_{44}$. 


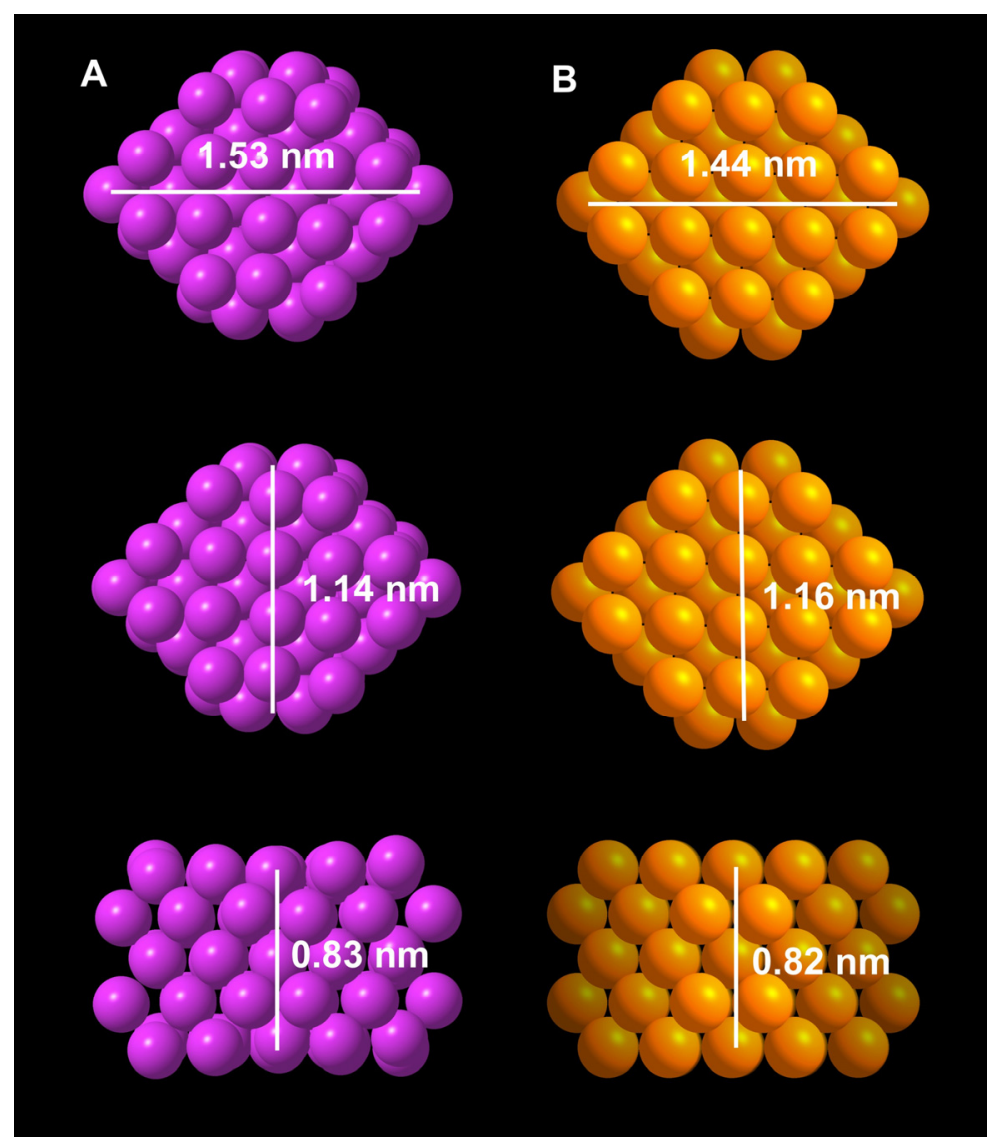

Figure S3. Comparison of the experimental $\mathrm{Au}_{84}$ kernel (A) with the $\mathrm{Au}_{84}$ kernel carved from an ideal face-centered cubic (fcc) lattice (B).

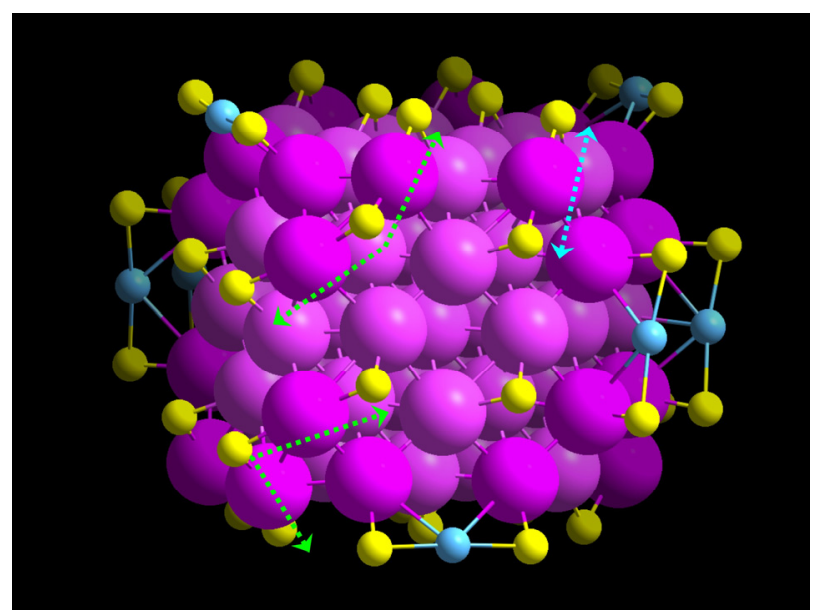

Figure S4. Edge and vertex protecting modes in the $\mathrm{Au}_{92}(\mathrm{TBBT})_{44}$ nanocrystal. At the edge, two bridging thiolates in the two joining facets give rise to a linear $-\mathrm{S}-\mathrm{Au}-\mathrm{S}-$ motif (blue dash line); at the vertex, three bridging thiolates in the three joining facets give rise to a $\mathrm{V}$-shaped $-\mathrm{S}-\mathrm{Au}-$ $\mathrm{S}-\mathrm{Au}-\mathrm{S}-$ motif (green dash lines). 


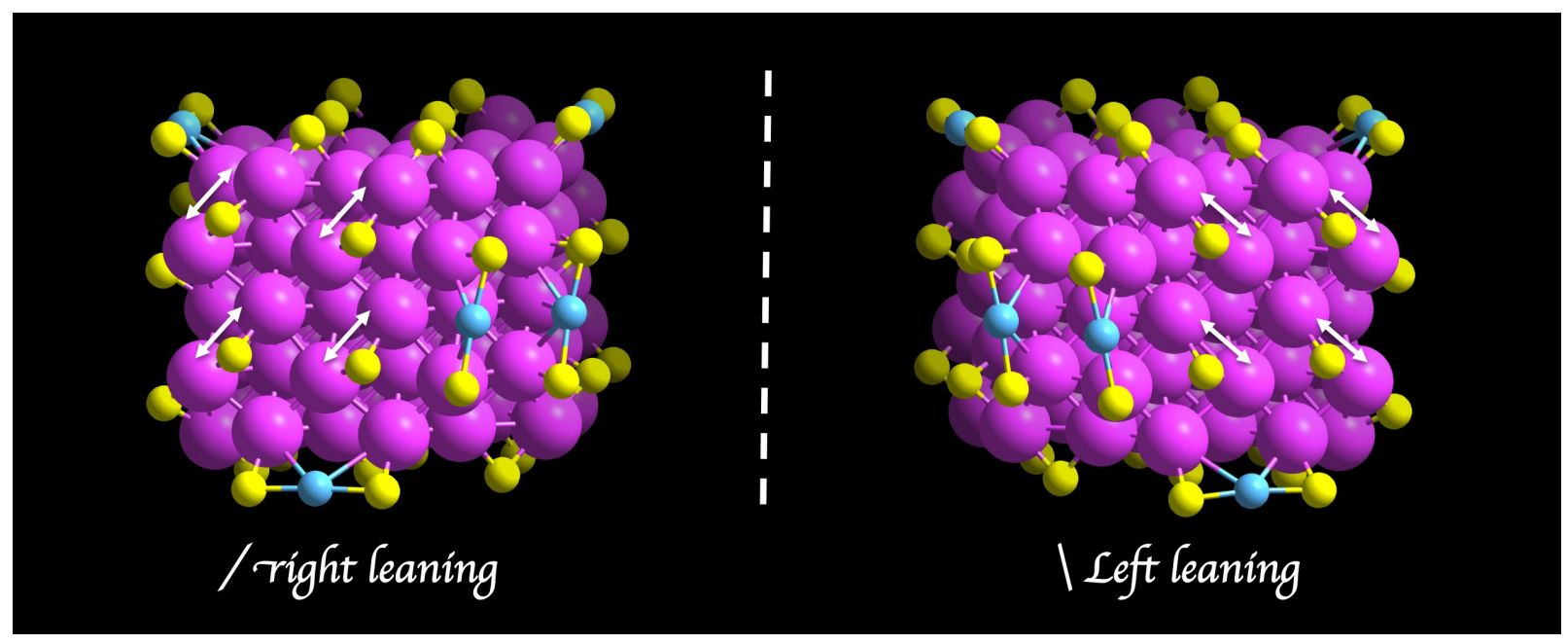

Figure S5. Chirality of the $\mathrm{Au}_{92}(\mathrm{TBBT})_{44}$ nanocrystal determined by the orientation of -SRbridging thiolates. Gold: magenta/blue. Sulfur: yellow. Carbon tails are omitted for clarity.

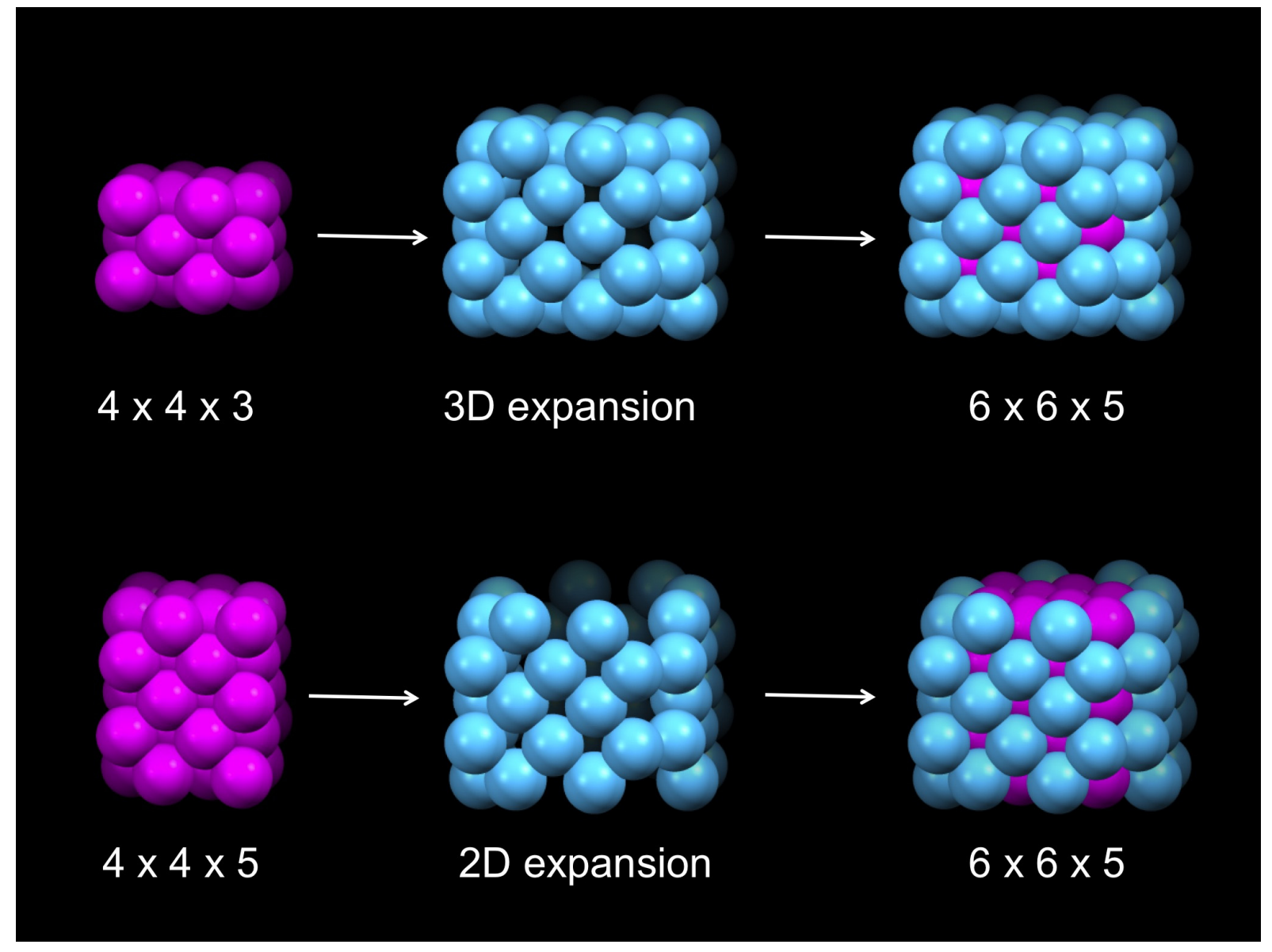

Figure S6. 3D expansion of the $4 \times 4 \times 3$ lattice in $\mathrm{Au}_{28}$ nanocrystal and $2 \mathrm{D}$ expansion of the $4 \times 4 \times 5$ lattice in $\mathrm{Au}_{44}$ nanocrystal. 
Table S1. Crystal data and structure refinement for $\mathrm{Au}_{92}(\mathrm{TBBT})_{44}$.

Identification code

Empirical formula

Formula weight

Temperature

Wavelength

Crystal system

Space group

Unit cell dimensions

Volume

Z

Density (calculated)

Absorption coefficient

$\mathrm{F}(000)$

Crystal size

Theta range for data collection

Index ranges

Reflections collected

Independent reflections

Completeness to theta $=51.35^{\circ}$

Absorption correction

Max. and min. transmission

Refinement method

Data / restraints / parameters

Goodness-of-fit on $\mathrm{F}^{2}$

Final $\mathrm{R}$ indices $[\mathrm{I}>2 \operatorname{sigma}(\mathrm{I})]$

$\mathrm{R}$ indices (all data)

Largest diff. peak and hole
$\mathrm{Au}_{92}(\mathrm{TBBT})_{44}$

C440 H572 Au92 S44

25392.54

230(2) K

$1.54178 \AA$

Monoclinic

$\mathrm{C} 2 / \mathrm{c}$

$\mathrm{a}=45.3000(11) \AA \quad \alpha=90^{\circ}$.

$\mathrm{b}=28.6539(7) \AA \quad \beta=90.940(2)^{\circ}$.

$\mathrm{c}=88.045(2) \AA \quad \gamma=90^{\circ}$.

$114269(5) \AA^{3}$

8

$2.952 \mathrm{Mg} / \mathrm{m}^{3}$

$44.901 \mathrm{~mm}^{-1}$

89472

$0.20 \times 0.12 \times 0.01 \mathrm{~mm}^{3}$

1.82 to $51.35^{\circ}$.

$-45<=\mathrm{h}<=45,-28<=\mathrm{k}<=28,-88<=\mathrm{l}<=88$

452199

$61863[\mathrm{R}(\mathrm{int})=0.1165]$

$99.5 \%$

Multi scan

0.6623 and 0.0403

Full-matrix least-squares on $\mathrm{F}^{2}$

61863 / 2207 / 3386

1.045

$\mathrm{R} 1=0.1080, \mathrm{wR} 2=0.3139$

$\mathrm{R} 1=0.1682, \mathrm{wR} 2=0.3737$

3.993 and -5.570 e. $\AA^{-3}$ 
Table S2. Atomic coordinates $\left(\times 10^{4}\right)$ and equivalent isotropic displacement parameters $\left(\AA^{2} \times\right.$ $10^{3}$ ) for $\mathrm{Au}_{92}(\mathrm{TBBT})_{44}$. U(eq) is defined as one third of the trace of the orthogonalized Uij tensor.

\begin{tabular}{|c|c|c|c|c|}
\hline & $\mathrm{x}$ & $\mathrm{y}$ & $\mathrm{z}$ & $\mathrm{U}(\mathrm{eq})$ \\
\hline $\mathrm{Au}(1)$ & $2727(1)$ & $4415(1)$ & $3878(1)$ & $36(1)$ \\
\hline $\mathrm{Au}(2)$ & $2685(1)$ & $3835(1)$ & $3612(1)$ & $37(1)$ \\
\hline $\mathrm{Au}(3)$ & $2249(1)$ & $4551(1)$ & $3668(1)$ & $37(1)$ \\
\hline $\mathrm{Au}(4)$ & $2257(1)$ & $3737(1)$ & $3860(1)$ & $38(1)$ \\
\hline $\mathrm{Au}(5)$ & $3154(1)$ & $4522(1)$ & $3633(1)$ & $40(1)$ \\
\hline $\mathrm{Au}(6)$ & $2311(1)$ & $4303(1)$ & $4129(1)$ & $39(1)$ \\
\hline $\mathrm{Au}(7)$ & $2716(1)$ & $5233(1)$ & $3683(1)$ & 41(1) \\
\hline $\mathrm{Au}(8)$ & 2741(1) & $3590(1)$ & $4071(1)$ & $39(1)$ \\
\hline $\mathrm{Au}(9)$ & 2294(1) & $5130(1)$ & $3936(1)$ & $41(1)$ \\
\hline $\mathrm{Au}(10)$ & $2666(1)$ & $4643(1)$ & $3416(1)$ & 41(1) \\
\hline $\operatorname{Au}(11)$ & 1821(1) & $4450(1)$ & $3917(1)$ & $41(1)$ \\
\hline $\mathrm{Au}(12)$ & 2201(1) & 3961(1) & $3399(1)$ & 41(1) \\
\hline $\mathrm{Au}(13)$ & $3195(1)$ & $5098(1)$ & 3899(1) & $43(1)$ \\
\hline $\mathrm{Au}(14)$ & $3117(1)$ & 3929(1) & $3369(1)$ & $44(1)$ \\
\hline $\mathrm{Au}(15)$ & 1781(1) & $3880(1)$ & $3647(1)$ & $43(1)$ \\
\hline $\mathrm{Au}(16)$ & 1811(1) & $5266(1)$ & $3725(1)$ & $46(1)$ \\
\hline $\mathrm{Au}(17)$ & $2204(1)$ & $3154(1)$ & 3599(1) & $44(1)$ \\
\hline $\operatorname{Au}(18)$ & $2780(1)$ & 4990(1) & $4148(1)$ & $42(1)$ \\
\hline $\mathrm{Au}(19)$ & $3164(1)$ & $3702(1)$ & $3827(1)$ & $42(1)$ \\
\hline $\operatorname{Au}(20)$ & $3210(1)$ & $4277(1)$ & 4094(1) & $43(1)$ \\
\hline $\operatorname{Au}(21)$ & $2715(1)$ & $3009(1)$ & $3804(1)$ & $46(1)$ \\
\hline $\operatorname{Au}(22)$ & $1826(1)$ & $3610(1)$ & $4102(1)$ & $46(1)$ \\
\hline $\operatorname{Au}(23)$ & $3133(1)$ & $5335(1)$ & $3433(1)$ & $49(1)$ \\
\hline $\operatorname{Au}(24)$ & $2231(1)$ & $5362(1)$ & $3474(1)$ & $46(1)$ \\
\hline $\operatorname{Au}(25)$ & 1763(1) & $4681(1)$ & $3455(1)$ & $46(1)$ \\
\hline $\operatorname{Au}(26)$ & 3133(1) & $3109(1)$ & $3584(1)$ & $48(1)$ \\
\hline $\operatorname{Au}(27)$ & 1344(1) & $4562(1)$ & 3693(1) & $51(1)$ \\
\hline $\operatorname{Au}(28)$ & $3590(1)$ & $3809(1)$ & $3573(1)$ & $50(1)$ \\
\hline $\operatorname{Au}(29)$ & $2243(1)$ & 2910(1) & $4047(1)$ & $49(1)$ \\
\hline $\mathrm{Au}(30)$ & $2286(1)$ & $3446(1)$ & $4311(1)$ & $51(1)$ \\
\hline $\operatorname{Au}(31)$ & $2808(1)$ & $4198(1)$ & 4339(1) & $50(1)$ \\
\hline $\operatorname{Au}(32)$ & $2288(1)$ & $5948(1)$ & $3733(1)$ & $51(1)$ \\
\hline
\end{tabular}




\begin{tabular}{|c|c|c|c|c|}
\hline $\mathrm{Au}(33)$ & 1886(1) & $5026(1)$ & $4190(1)$ & $48(1)$ \\
\hline $\mathrm{Au}(34)$ & $2758(1)$ & $5808(1)$ & $3960(1)$ & $50(1)$ \\
\hline $\mathrm{Au}(35)$ & $2656(1)$ & $4033(1)$ & $3152(1)$ & $50(1)$ \\
\hline $\mathrm{Au}(36)$ & $3629(1)$ & $4352(1)$ & $3856(1)$ & $51(1)$ \\
\hline $\operatorname{Au}(37)$ & $1344(1)$ & $3749(1)$ & $3905(1)$ & $51(1)$ \\
\hline $\mathrm{Au}(38)$ & $3617(1)$ & $5228(1)$ & $3638(1)$ & $52(1)$ \\
\hline $\mathrm{Au}(39)$ & $2355(1)$ & 4899(1) & $4400(1)$ & $53(1)$ \\
\hline $\mathrm{Au}(40)$ & $2675(1)$ & $5463(1)$ & $3222(1)$ & $53(1)$ \\
\hline $\mathrm{Au}(41)$ & $1408(1)$ & $4279(1)$ & $4159(1)$ & $54(1)$ \\
\hline $\mathrm{Au}(42)$ & $2164(1)$ & $4804(1)$ & $3212(1)$ & $52(1)$ \\
\hline $\mathrm{Au}(43)$ & $3090(1)$ & $4797(1)$ & $3174(1)$ & $54(1)$ \\
\hline $\mathrm{Au}(44)$ & $1905(1)$ & $4264(1)$ & $4378(1)$ & $53(1)$ \\
\hline $\mathrm{Au}(45)$ & $3579(1)$ & $4557(1)$ & $3385(1)$ & $53(1)$ \\
\hline $\mathrm{Au}(46)$ & $1766(1)$ & $3069(1)$ & $3804(1)$ & $53(1)$ \\
\hline $\mathrm{Au}(47)$ & $2665(1)$ & $3254(1)$ & $3337(1)$ & $52(1)$ \\
\hline $\mathrm{Au}(48)$ & $3191(1)$ & $5912(1)$ & $3687(1)$ & $55(1)$ \\
\hline $\mathrm{Au}(49)$ & $3619(1)$ & $3570(1)$ & $4033(1)$ & $56(1)$ \\
\hline $\mathrm{Au}(50)$ & $1400(1)$ & $5182(1)$ & 3994(1) & $56(1)$ \\
\hline $\mathrm{Au}(51)$ & $3165(1)$ & $2870(1)$ & $4024(1)$ & $53(1)$ \\
\hline $\mathrm{Au}(52)$ & $1769(1)$ & $5493(1)$ & $3275(1)$ & $58(1)$ \\
\hline $\mathrm{Au}(53)$ & $1324(1)$ & $3984(1)$ & $3445(1)$ & $56(1)$ \\
\hline $\mathrm{Au}(54)$ & $3665(1)$ & $5007(1)$ & $4118(1)$ & $57(1)$ \\
\hline $\mathrm{Au}(55)$ & $2145(1)$ & $3358(1)$ & $3149(1)$ & $59(1)$ \\
\hline $\mathrm{Au}(56)$ & $1847(1)$ & $5847(1)$ & $4010(1)$ & $58(1)$ \\
\hline $\mathrm{Au}(57)$ & $1789(1)$ & $6073(1)$ & $3543(1)$ & $60(1)$ \\
\hline $\mathrm{Au}(58)$ & $3218(1)$ & $3404(1)$ & $4278(1)$ & $57(1)$ \\
\hline $\mathrm{Au}(59)$ & $1754(1)$ & $3274(1)$ & $3378(1)$ & $55(1)$ \\
\hline $\mathrm{Au}(60)$ & $2756(1)$ & $2765(1)$ & $4243(1)$ & $56(1)$ \\
\hline $\mathrm{Au}(61)$ & $2382(1)$ & $5703(1)$ & $4195(1)$ & $57(1)$ \\
\hline $\mathrm{Au}(62)$ & $1841(1)$ & $2788(1)$ & $4271(1)$ & $59(1)$ \\
\hline $\mathrm{Au}(63)$ & $2639(1)$ & $6063(1)$ & $3484(1)$ & $59(1)$ \\
\hline $\mathrm{Au}(64)$ & $3085(1)$ & $3364(1)$ & $3117(1)$ & $58(1)$ \\
\hline $\operatorname{Au}(65)$ & 1713(1) & $4030(1)$ & $3190(1)$ & $58(1)$ \\
\hline $\mathrm{Au}(66)$ & $3249(1)$ & $5684(1)$ & $4156(1)$ & $57(1)$ \\
\hline $\mathrm{Au}(67)$ & $3239(1)$ & $4863(1)$ & $4352(1)$ & $57(1)$ \\
\hline $\mathrm{Au}(68)$ & $1336(1)$ & $5444(1)$ & $3513(1)$ & $62(1)$ \\
\hline $\mathrm{Au}(69)$ & $3639(1)$ & $5777(1)$ & $3925(1)$ & $60(1)$ \\
\hline $\mathrm{Au}(70)$ & $3571(1)$ & $6007(1)$ & $3452(1)$ & $61(1)$ \\
\hline
\end{tabular}




\begin{tabular}{|c|c|c|c|c|}
\hline $\operatorname{Au}(71)$ & $1404(1)$ & 5981(1) & $3775(1)$ & $64(1)$ \\
\hline $\operatorname{Au}(72)$ & $1229(1)$ & $3110(1)$ & $3598(1)$ & $64(1)$ \\
\hline $\operatorname{Au}(73)$ & $3673(1)$ & 2904(1) & 3784(1) & $64(1)$ \\
\hline $\operatorname{Au}(74)$ & $3568(1)$ & $4015(1)$ & $3096(1)$ & $64(1)$ \\
\hline $\operatorname{Au}(75)$ & $3623(1)$ & $5446(1)$ & $3176(1)$ & $66(1)$ \\
\hline $\operatorname{Au}(76)$ & $1488(1)$ & $5709(1)$ & $4255(1)$ & $66(1)$ \\
\hline $\operatorname{Au}(77)$ & $2164(1)$ & $6276(1)$ & $3243(1)$ & $65(1)$ \\
\hline $\mathrm{Au}(78)$ & $3591(1)$ & $3057(1)$ & $3313(1)$ & $70(1)$ \\
\hline $\mathrm{Au}(79)$ & $3120(1)$ & $6266(1)$ & $3234(1)$ & $71(1)$ \\
\hline $\mathrm{Au}(80)$ & $1414(1)$ & 4931(1) & $4459(1)$ & $71(1)$ \\
\hline $\mathrm{Au}(81)$ & $1410(1)$ & $3447(1)$ & 4387(1) & $72(1)$ \\
\hline $\mathrm{Au}(82)$ & $1336(1)$ & $2803(1)$ & $4070(1)$ & $73(1)$ \\
\hline $\mathrm{Au}(83)$ & $2924(1)$ & $5671(1)$ & $4464(1)$ & $74(1)$ \\
\hline $\mathrm{Au}(84)$ & 1981(1) & $5726(1)$ & $4488(1)$ & $79(1)$ \\
\hline $\mathrm{Au}(85)$ & $2634(1)$ & 2891(1) & $2916(1)$ & $77(1)$ \\
\hline $\mathrm{Au}(86)$ & $4065(1)$ & 4164(1) & 4195(1) & $81(1)$ \\
\hline $\mathrm{Au}(87)$ & $2261(1)$ & 2173(1) & $4421(1)$ & $81(1)$ \\
\hline $\mathrm{Au}(88)$ & $1478(1)$ & 4912(1) & $3036(1)$ & $78(1)$ \\
\hline $\mathrm{Au}(89)$ & $4011(1)$ & 6388(1) & $3670(1)$ & $84(1)$ \\
\hline $\operatorname{Au}(90)$ & $3501(1)$ & 4004(1) & $4522(1)$ & $81(1)$ \\
\hline $\mathrm{Au}(91)$ & $911(1)$ & $4724(1)$ & $3367(1)$ & $90(1)$ \\
\hline $\mathrm{Au}(92)$ & 1092(1) & 6392(1) & $4086(1)$ & $96(1)$ \\
\hline$S(1)$ & $4008(2)$ & 4900(4) & $3796(2)$ & $58(3)$ \\
\hline $\mathrm{S}(2)$ & $2670(3)$ & $3571(4)$ & $4498(1)$ & $59(3)$ \\
\hline$S(3)$ & $1040(3)$ & $4679(5)$ & 4299(2) & $75(4)$ \\
\hline$S(4)$ & $2396(2)$ & $2362(4)$ & $3866(1)$ & $57(3)$ \\
\hline$S(5)$ & $926(3)$ & $3562(5)$ & $3745(2)$ & $67(4)$ \\
\hline$S(6)$ & $2273(3)$ & $4353(5)$ & 2993(1) & $64(4)$ \\
\hline$S(7)$ & $2488(3)$ & 2593(4) & $3468(1)$ & $58(3)$ \\
\hline$S(8)$ & $3987(3)$ & $5039(5)$ & $3312(2)$ & $61(3)$ \\
\hline$S(9)$ & 1979(3) & $6754(5)$ & $3430(2)$ & $61(3)$ \\
\hline$S(10)$ & 993(3) & $5054(5)$ & $3819(2)$ & $68(4)$ \\
\hline $\mathrm{S}(11)$ & 2151(3) & $6486(5)$ & $3934(2)$ & $70(4)$ \\
\hline$S(12)$ & $3929(3)$ & $3569(5)$ & $3211(2)$ & $70(4)$ \\
\hline$S(13)$ & 2334(3) & $5838(5)$ & $3047(2)$ & $72(4)$ \\
\hline$S(14)$ & $2125(3)$ & $2848(5)$ & $2937(2)$ & $68(4)$ \\
\hline$S(15)$ & 2934(3) & $6737(5)$ & $3423(2)$ & $69(4)$ \\
\hline$S(16)$ & $4007(3)$ & $3434(5)$ & $3696(2)$ & $69(4)$ \\
\hline
\end{tabular}




\begin{tabular}{|c|c|c|c|c|}
\hline $\mathrm{S}(17)$ & $4028(3)$ & $6331(5)$ & $3928(2)$ & $69(4)$ \\
\hline $\mathrm{S}(18)$ & $3373(3)$ & $2303(5)$ & $3867(2)$ & $72(4)$ \\
\hline $\mathrm{S}(19)$ & $3242(3)$ & $4430(5)$ & $2945(2)$ & $64(4)$ \\
\hline $\mathrm{S}(20)$ & $1757(4)$ & $2182(5)$ & $4450(2)$ & $80(4)$ \\
\hline $\mathrm{S}(21)$ & $2755(3)$ & $2080(5)$ & 4394(2) & $75(4)$ \\
\hline $\mathrm{S}(22)$ & $1765(3)$ & $3697(5)$ & $4560(2)$ & $70(4)$ \\
\hline $\mathrm{S}(23)$ & 3999(3) & $3369(5)$ & $4211(2)$ & $76(4)$ \\
\hline $\mathrm{S}(24)$ & $3398(3)$ & $3214(6)$ & $4530(2)$ & $81(4)$ \\
\hline $\mathrm{S}(25)$ & $932(3)$ & $3985(6)$ & $3260(2)$ & $79(4)$ \\
\hline $\mathrm{S}(26)$ & $3336(3)$ & $5934(5)$ & $3028(2)$ & $67(4)$ \\
\hline $\mathrm{S}(27)$ & $2700(3)$ & $5109(5)$ & $4600(2)$ & $75(4)$ \\
\hline $\mathrm{S}(28)$ & $1375(3)$ & $5671(6)$ & $3103(2)$ & $79(4)$ \\
\hline $\mathrm{S}(29)$ & $3974(3)$ & $6518(5)$ & $3412(2)$ & $82(5)$ \\
\hline $\mathrm{S}(30)$ & $3151(3)$ & $2868(5)$ & 2901(2) & $80(4)$ \\
\hline $\mathrm{S}(31)$ & $3122(3)$ & $6284(5)$ & $4332(2)$ & $74(4)$ \\
\hline $\mathrm{S}(32)$ & $1620(3)$ & $2346(5)$ & $3913(2)$ & $77(4)$ \\
\hline $\mathrm{S}(33)$ & $3632(3)$ & $4784(6)$ & $4535(2)$ & $81(4)$ \\
\hline $\mathrm{S}(34)$ & $820(3)$ & $5469(6)$ & $3449(2)$ & $83(5)$ \\
\hline$S(35)$ & $1732(3)$ & $5227(5)$ & $4642(2)$ & $79(4)$ \\
\hline$S(36)$ & 1494(3) & $2611(5)$ & $3452(2)$ & $74(4)$ \\
\hline $\mathrm{S}(37)$ & $2935(4)$ & $6480(5)$ & $3826(2)$ & $98(6)$ \\
\hline $\mathrm{S}(38)$ & $1047(3)$ & $6546(5)$ & $3832(2)$ & $93(5)$ \\
\hline $\mathrm{S}(39)$ & 1533(3) & $4177(5)$ & $2934(2)$ & $78(4)$ \\
\hline $\mathrm{S}(40)$ & $4179(3)$ & $4942(6)$ & $4190(2)$ & $84(5)$ \\
\hline $\mathrm{S}(41)$ & $3237(3)$ & $2525(5)$ & $3393(2)$ & $85(5)$ \\
\hline $\mathrm{S}(42)$ & 1032(3) & $3087(6)$ & $4253(2)$ & $98(5)$ \\
\hline $\mathrm{S}(43)$ & $1153(4)$ & $6282(6)$ & $4340(2)$ & $104(6)$ \\
\hline $\mathrm{S}(44)$ & $2312(4)$ & $6267(5)$ & $4394(2)$ & $100(6)$ \\
\hline$C(1)$ & $4322(5)$ & $4646(11)$ & $3726(4)$ & $66(10)$ \\
\hline $\mathrm{C}(2)$ & $4470(6)$ & $4855(10)$ & $3607(4)$ & $83(11)$ \\
\hline$C(3)$ & $4751(6)$ & $4705(10)$ & $3569(3)$ & $84(12)$ \\
\hline$C(4)$ & $4886(5)$ & $4347(9)$ & $3651(3)$ & $95(12)$ \\
\hline$C(5)$ & $4738(6)$ & $4138(9)$ & $3771(3)$ & $83(11)$ \\
\hline$C(6)$ & $4457(6)$ & $4287(11)$ & $3808(3)$ & $81(11)$ \\
\hline$C(7)$ & $5176(6)$ & $4162(13)$ & $3619(4)$ & $170(40)$ \\
\hline$C(8)$ & $5340(7)$ & $4467(18)$ & $3501(5)$ & $300(70)$ \\
\hline$C(9)$ & $5418(6)$ & $4249(18)$ & $3737(6)$ & $170(40)$ \\
\hline$C(10)$ & $5180(9)$ & $3654(14)$ & $3553(6)$ & $240(60)$ \\
\hline
\end{tabular}




\begin{tabular}{|c|c|c|c|c|}
\hline $\mathrm{C}(11)$ & $2586(8)$ & $3751(11)$ & $4687(2)$ & $82(12)$ \\
\hline$C(12)$ & 2779(7) & $4066(12)$ & $4756(3)$ & $91(13)$ \\
\hline$C(13)$ & $2768(6)$ & $4141(10)$ & 4912(3) & $98(13)$ \\
\hline$C(14)$ & $2565(5)$ & $3901(8)$ & 4999(2) & $95(13)$ \\
\hline$C(15)$ & $2372(6)$ & $3586(10)$ & $4930(3)$ & $97(13)$ \\
\hline$C(16)$ & $2382(7)$ & $3511(11)$ & $4774(3)$ & $88(12)$ \\
\hline$C(17)$ & $2541(7)$ & $3950(11)$ & $5162(2)$ & $88(18)$ \\
\hline $\mathrm{C}(18)$ & $2731(10)$ & $4362(13)$ & $5224(3)$ & $180(40)$ \\
\hline $\mathrm{C}(19)$ & $2262(9)$ & $4176(16)$ & $5221(3)$ & $240(50)$ \\
\hline$C(20)$ & $2640(10)$ & $3513(13)$ & $5257(3)$ & $120(20)$ \\
\hline$C(21)$ & $834(8)$ & $4301(12)$ & $4408(5)$ & $109(15)$ \\
\hline $\mathrm{C}(22)$ & $636(9)$ & $4032(15)$ & $4323(4)$ & $123(16)$ \\
\hline$C(23)$ & $438(8)$ & $3741(13)$ & 4397(4) & 134(16) \\
\hline$C(24)$ & $439(7)$ & $3721(11)$ & $4555(4)$ & $135(17)$ \\
\hline$C(25)$ & $637(8)$ & $3990(14)$ & $4639(4)$ & $128(16)$ \\
\hline$C(26)$ & $835(8)$ & $4281(14)$ & $4565(5)$ & 123(16) \\
\hline$C(27)$ & $238(10)$ & $3435(17)$ & $4641(6)$ & $250(60)$ \\
\hline $\mathrm{C}(28)$ & $60(12)$ & $3093(19)$ & $4537(8)$ & $430(120)$ \\
\hline$C(29)$ & $370(15)$ & $3029(19)$ & $4729(8)$ & $490(140)$ \\
\hline$C(30)$ & $1(11)$ & $3710(20)$ & $4733(7)$ & $390(100)$ \\
\hline $\mathrm{C}(31)$ & $2521(8)$ & $1838(9)$ & $3968(3)$ & $68(10)$ \\
\hline $\mathrm{C}(32)$ & $2787(7)$ & $1625(11)$ & $3932(3)$ & $85(12)$ \\
\hline$C(33)$ & $2877(6)$ & $1226(11)$ & $4009(4)$ & $97(13)$ \\
\hline$C(34)$ & $2703(6)$ & $1040(9)$ & $4122(3)$ & $102(13)$ \\
\hline$C(35)$ & $2437(6)$ & $1253(10)$ & $4158(3)$ & $97(13)$ \\
\hline$C(36)$ & $2346(7)$ & $1652(10)$ & 4081(4) & $81(12)$ \\
\hline$C(37)$ & $2777(9)$ & $620(11)$ & $4207(4)$ & $220(40)$ \\
\hline$C(38)$ & $3097(10)$ & $455(15)$ & $4176(6)$ & $290(60)$ \\
\hline$C(39)$ & $2849(13)$ & $678(17)$ & $4375(4)$ & $320(60)$ \\
\hline $\mathrm{C}(40)$ & $2575(12)$ & $188(10)$ & $4173(6)$ & $260(50)$ \\
\hline $\mathrm{C}(41)$ & $683(7)$ & $3185(11)$ & $3823(4)$ & $88(12)$ \\
\hline $\mathrm{C}(42)$ & $568(8)$ & $2853(12)$ & $3723(3)$ & $91(12)$ \\
\hline$C(43)$ & $356(7)$ & $2538(10)$ & $3773(3)$ & $87(12)$ \\
\hline $\mathrm{C}(44)$ & $260(6)$ & 2556(9) & $3922(3)$ & $93(13)$ \\
\hline $\mathrm{C}(45)$ & $374(8)$ & $2888(12)$ & 4021(3) & $100(13)$ \\
\hline $\mathrm{C}(46)$ & $586(8)$ & $3203(11)$ & $3972(4)$ & $88(12)$ \\
\hline $\mathrm{C}(47)$ & $37(8)$ & $2247(14)$ & $3982(5)$ & $200(40)$ \\
\hline$C(48)$ & $-41(12)$ & $1844(15)$ & $3871(7)$ & $300(60)$ \\
\hline
\end{tabular}




\begin{tabular}{|c|c|c|c|c|}
\hline$C(49)$ & $137(13)$ & $1900(17)$ & $4103(6)$ & $530(160)$ \\
\hline$C(50)$ & $-265(8)$ & $2490(20)$ & $4020(7)$ & $320(60)$ \\
\hline$C(51)$ & $2367(8)$ & $4671(10)$ & $2830(3)$ & $67(11)$ \\
\hline$C(52)$ & $2183(7)$ & $5028(11)$ & 2779(3) & $81(11)$ \\
\hline$C(53)$ & $2223(6)$ & $5222(9)$ & $2636(3)$ & $85(12)$ \\
\hline$C(54)$ & $2447(5)$ & $5060(8)$ & $2544(2)$ & $100(13)$ \\
\hline$C(55)$ & $2632(6)$ & $4702(10)$ & $2595(3)$ & $95(13)$ \\
\hline$C(56)$ & $2592(7)$ & $4508(10)$ & $2738(3)$ & $84(12)$ \\
\hline$C(57)$ & $2502(8)$ & $5235(12)$ & 2393(3) & $120(20)$ \\
\hline$C(58)$ & $2318(11)$ & $5680(14)$ & $2357(4)$ & $220(50)$ \\
\hline$C(59)$ & 2788(9) & $5502(16)$ & $2370(4)$ & $200(40)$ \\
\hline$C(60)$ & $2430(11)$ & $4886(16)$ & $2260(2)$ & $180(40)$ \\
\hline$C(61)$ & $2293(7)$ & 2198(9) & $3349(3)$ & $63(10)$ \\
\hline$C(62)$ & $2365(7)$ & $2115(11)$ & $3199(3)$ & $75(11)$ \\
\hline$C(63)$ & $2205(8)$ & 1791(11) & $3114(3)$ & $96(12)$ \\
\hline$C(64)$ & 1974(7) & 1549(9) & $3179(3)$ & $81(12)$ \\
\hline$C(65)$ & 1902(6) & 1631(9) & $3330(3)$ & $78(11)$ \\
\hline$C(66)$ & $2062(8)$ & $1956(10)$ & $3415(3)$ & 71(11) \\
\hline$C(67)$ & $1792(10)$ & $1210(14)$ & $3099(5)$ & $410(70)$ \\
\hline$C(68)$ & $1919(14)$ & 1087(19) & $2941(5)$ & $430(80)$ \\
\hline$C(69)$ & $1819(13)$ & $705(12)$ & $3149(6)$ & $510(90)$ \\
\hline$C(70)$ & 1464(9) & $1360(20)$ & $3067(7)$ & $490(90)$ \\
\hline $\mathrm{C}(71)$ & $4251(7)$ & $4803(14)$ & $3183(4)$ & $107(14)$ \\
\hline$C(72)$ & $4446(8)$ & $4450(14)$ & $3225(3)$ & $111(14)$ \\
\hline$C(73)$ & $4691(7)$ & $4360(12)$ & $3136(4)$ & $119(15)$ \\
\hline$C(74)$ & $4740(6)$ & $4622(10)$ & $3007(3)$ & $124(15)$ \\
\hline$C(75)$ & $4545(7)$ & $4975(12)$ & $2965(3)$ & $124(15)$ \\
\hline$C(76)$ & $4300(7)$ & $5066(14)$ & $3053(4)$ & $109(14)$ \\
\hline$C(77)$ & 4992(7) & $4559(15)$ & 2909(4) & $180(40)$ \\
\hline$C(78)$ & $5169(9)$ & $4111(17)$ & $2951(6)$ & $200(40)$ \\
\hline$C(79)$ & $4930(11)$ & $4370(20)$ & $2750(4)$ & $270(70)$ \\
\hline $\mathrm{C}(80)$ & $5223(8)$ & 4966(19) & 2912(6) & $240(60)$ \\
\hline$C(81)$ & $1686(6)$ & $7075(11)$ & $3342(4)$ & $89(12)$ \\
\hline$C(82)$ & $1746(6)$ & $7386(12)$ & $3225(5)$ & $96(13)$ \\
\hline$C(83)$ & $1525(8)$ & $7677(12)$ & $3169(4)$ & $106(14)$ \\
\hline$C(84)$ & $1243(7)$ & $7658(11)$ & $3229(4)$ & $109(14)$ \\
\hline$C(85)$ & $1183(6)$ & $7347(13)$ & $3346(4)$ & $112(14)$ \\
\hline$C(86)$ & $1404(7)$ & $7056(12)$ & $3402(4)$ & $103(13)$ \\
\hline
\end{tabular}




\begin{tabular}{|c|c|c|c|c|}
\hline $\mathrm{C}(87)$ & $1000(9)$ & 7953(17) & $3179(7)$ & $530(90)$ \\
\hline $\mathrm{C}(88)$ & $1076(14)$ & $8230(20)$ & $3033(7)$ & $540(100)$ \\
\hline$C(89)$ & $743(10)$ & $7720(30)$ & $3098(8)$ & $570(110)$ \\
\hline $\mathrm{C}(90)$ & $900(13)$ & $8328(18)$ & $3297(9)$ & $560(100)$ \\
\hline$C(91)$ & $680(5)$ & 4764(11) & $3877(4)$ & $69(11)$ \\
\hline$C(92)$ & $540(7)$ & $4475(13)$ & $3771(3)$ & $93(12)$ \\
\hline $\mathrm{C}(93)$ & $261(7)$ & $4298(11)$ & $3800(3)$ & $99(13)$ \\
\hline$C(94)$ & $122(5)$ & $4409(9)$ & $3935(3)$ & $95(13)$ \\
\hline$C(95)$ & $262(6)$ & $4698(11)$ & 4041(3) & $92(12)$ \\
\hline$C(96)$ & $541(6)$ & $4876(11)$ & $4011(4)$ & $81(11)$ \\
\hline$C(97)$ & $-170(6)$ & $4248(14)$ & $3975(5)$ & $150(30)$ \\
\hline $\mathrm{C}(98)$ & $-286(8)$ & $3870(17)$ & $3862(6)$ & $300(70)$ \\
\hline $\mathrm{C}(99)$ & $-191(9)$ & $3908(17)$ & $4106(6)$ & $260(60)$ \\
\hline$C(100)$ & $-415(5)$ & 4634(19) & $3976(6)$ & $300(70)$ \\
\hline $\mathrm{C}(101)$ & $1930(12)$ & $6957(13)$ & $3873(6)$ & $143(18)$ \\
\hline$C(102)$ & 1661(12) & $7085(14)$ & $3935(5)$ & 141(17) \\
\hline$C(103)$ & 1522(9) & $7494(15)$ & $3887(5)$ & $146(18)$ \\
\hline$C(104)$ & $1653(9)$ & $7775(12)$ & $3779(5)$ & $155(18)$ \\
\hline $\mathrm{C}(105)$ & $1922(10)$ & 7647(15) & $3718(5)$ & 161(18) \\
\hline$C(106)$ & $2060(10)$ & $7238(17)$ & $3765(6)$ & $154(18)$ \\
\hline $\mathrm{C}(107)$ & $1523(14)$ & $8203(16)$ & $3721(7)$ & $380(100)$ \\
\hline$C(108)$ & $1250(15)$ & $8350(20)$ & $3813(10)$ & $490(140)$ \\
\hline C(109) & $1680(19)$ & $8656(12)$ & $3761(10)$ & $500(150)$ \\
\hline$C(110)$ & 1414(19) & $8180(30)$ & $3551(8)$ & $600(190)$ \\
\hline $\mathrm{C}(111)$ & $4079(7)$ & $3279(15)$ & $3050(3)$ & $99(14)$ \\
\hline$C(112)$ & $4236(8)$ & $3560(12)$ & 2951(4) & $109(14)$ \\
\hline$C(113)$ & $4358(8)$ & $3368(13)$ & $2821(4)$ & $124(15)$ \\
\hline$C(114)$ & $4324(7)$ & $2895(14)$ & 2791(3) & $125(16)$ \\
\hline$C(115)$ & $4167(8)$ & $2614(12)$ & $2890(4)$ & $120(15)$ \\
\hline$C(116)$ & $4045(8)$ & $2806(15)$ & $3019(4)$ & $106(14)$ \\
\hline$C(117)$ & $4439(11)$ & $2667(19)$ & $2657(4)$ & $330(80)$ \\
\hline $\mathrm{C}(118)$ & $4649(13)$ & $2990(30)$ & $2568(5)$ & $700(200)$ \\
\hline$C(119)$ & $4685(13)$ & $2320(20)$ & $2682(7)$ & $330(80)$ \\
\hline$C(120)$ & $4200(15)$ & $2500(30)$ & $2538(4)$ & $700(200)$ \\
\hline $\mathrm{C}(121)$ & $2517(8)$ & $6213(13)$ & 2915(4) & $111(15)$ \\
\hline$C(122)$ & 2742(9) & $6051(11)$ & 2824(4) & $119(15)$ \\
\hline$C(123)$ & $2858(7)$ & $6339(11)$ & 2713(4) & $119(15)$ \\
\hline $\mathrm{C}(124)$ & $2750(6)$ & $6789(10)$ & 2693(3) & $114(15)$ \\
\hline
\end{tabular}




\begin{tabular}{|c|c|c|c|c|}
\hline$C(125)$ & $2524(7)$ & $6951(11)$ & $2784(4)$ & $130(16)$ \\
\hline$C(126)$ & $2408(8)$ & $6663(14)$ & $2895(4)$ & $124(15)$ \\
\hline$C(127)$ & 2861(8) & $7116(12)$ & $2582(4)$ & $110(20)$ \\
\hline$C(128)$ & $3076(11)$ & $6878(17)$ & $2470(5)$ & $270(70)$ \\
\hline$C(129)$ & $2654(11)$ & $7248(18)$ & $2452(4)$ & $250(60)$ \\
\hline$C(130)$ & $3034(11)$ & $7544(13)$ & $2650(6)$ & $230(50)$ \\
\hline$C(131)$ & $1966(8)$ & $3167(11)$ & 2783(3) & $89(12)$ \\
\hline$C(132)$ & 1674(8) & $3079(10)$ & 2741(3) & $91(12)$ \\
\hline$C(133)$ & $1537(6)$ & $3346(9)$ & 2628(3) & $95(13)$ \\
\hline$C(134)$ & $1693(6)$ & $3701(8)$ & $2557(3)$ & $93(13)$ \\
\hline$C(135)$ & 1984(6) & $3789(10)$ & 2599(3) & $93(12)$ \\
\hline$C(136)$ & 2121(6) & $3522(12)$ & 2712(4) & $85(12)$ \\
\hline$C(137)$ & $1570(7)$ & $3991(11)$ & $2437(3)$ & $150(30)$ \\
\hline$C(138)$ & $1232(7)$ & $3917(15)$ & 2416(4) & $120(20)$ \\
\hline$C(139)$ & $1523(10)$ & $4503(11)$ & $2473(5)$ & $180(40)$ \\
\hline$C(140)$ & $1704(10)$ & $3910(17)$ & $2276(3)$ & $240(60)$ \\
\hline$C(141)$ & 2744(9) & 7197(11) & $3330(5)$ & $116(15)$ \\
\hline$C(142)$ & $2569(9)$ & $7507(13)$ & $3410(4)$ & $122(15)$ \\
\hline$C(143)$ & $2470(8)$ & $7916(12)$ & $3342(4)$ & $129(16)$ \\
\hline$C(144)$ & $2545(7)$ & $8015(10)$ & 3193(4) & $146(17)$ \\
\hline$C(145)$ & 2719(9) & $7705(13)$ & $3112(4)$ & $150(17)$ \\
\hline$C(146)$ & 2819(10) & 7296(13) & 3181(5) & $139(16)$ \\
\hline$C(147)$ & $2448(11)$ & $8428(13)$ & $3111(5)$ & $270(60)$ \\
\hline$C(148)$ & $2297(15)$ & $8785(14)$ & $3218(7)$ & $430(120)$ \\
\hline$C(149)$ & $2682(15)$ & $8784(14)$ & $3071(8)$ & $280(70)$ \\
\hline$C(150)$ & $2220(14)$ & $8340(20)$ & 2978(6) & $350(90)$ \\
\hline$C(151)$ & $4209(7)$ & $3134(12)$ & $3562(4)$ & $90(12)$ \\
\hline$C(152)$ & $4394(8)$ & $3396(9)$ & $3471(4)$ & $94(13)$ \\
\hline$C(153)$ & $4589(7)$ & $3174(10)$ & $3374(4)$ & $109(14)$ \\
\hline$C(154)$ & $4600(6)$ & $2689(10)$ & $3370(3)$ & $120(14)$ \\
\hline$C(155)$ & $4416(7)$ & $2427(9)$ & $3461(4)$ & $123(14)$ \\
\hline$C(156)$ & $4220(7)$ & $2649(11)$ & $3557(4)$ & $102(13)$ \\
\hline$C(157)$ & 4793(8) & $2427(14)$ & $3271(4)$ & $160(30)$ \\
\hline$C(158)$ & $5022(10)$ & 2747(19) & $3193(5)$ & $290(70)$ \\
\hline$C(159)$ & $5039(9)$ & $2148(17)$ & $3347(6)$ & $220(50)$ \\
\hline$C(160)$ & $4634(12)$ & 2155(19) & $3139(5)$ & $270(60)$ \\
\hline$C(161)$ & $4345(5)$ & $6026(10)$ & $3986(4)$ & $75(11)$ \\
\hline$C(162)$ & $4441(6)$ & $6062(10)$ & 4137(4) & $87(12)$ \\
\hline
\end{tabular}




\begin{tabular}{|c|c|c|c|c|}
\hline$C(163)$ & $4686(6)$ & $5810(10)$ & $4187(3)$ & $93(12)$ \\
\hline$C(164)$ & $4835(5)$ & $5522(8)$ & $4087(3)$ & $98(13)$ \\
\hline$C(165)$ & $4739(6)$ & $5485(9)$ & $3937(3)$ & $94(12)$ \\
\hline$C(166)$ & $4494(6)$ & $5737(11)$ & $3886(3)$ & $77(11)$ \\
\hline$C(167)$ & $5096(6)$ & $5253(10)$ & $4128(4)$ & $130(30)$ \\
\hline$C(168)$ & $5155(8)$ & $5257(15)$ & $4302(4)$ & $170(40)$ \\
\hline$C(169)$ & $5064(8)$ & $4726(10)$ & $4130(5)$ & $130(30)$ \\
\hline$C(170)$ & $5390(5)$ & $5421(15)$ & $4055(5)$ & $150(30)$ \\
\hline$C(171)$ & $3605(7)$ & 1923(11) & $3970(4)$ & $84(12)$ \\
\hline$C(172)$ & $3838(8)$ & 1711(13) & $3896(3)$ & $93(13)$ \\
\hline$C(173)$ & $4002(7)$ & $1365(12)$ & $3969(4)$ & $102(13)$ \\
\hline$C(174)$ & $3933(7)$ & $1231(11)$ & $4116(4)$ & $105(14)$ \\
\hline$C(175)$ & $3699(8)$ & $1442(12)$ & $4190(3)$ & 101(13) \\
\hline$C(176)$ & $3535(7)$ & $1788(12)$ & $4117(4)$ & $90(13)$ \\
\hline$C(177)$ & $4088(11)$ & $868(16)$ & $4200(5)$ & $490(80)$ \\
\hline$C(178)$ & $4372(12)$ & $710(20)$ & $4118(7)$ & $470(90)$ \\
\hline$C(179)$ & $4269(13)$ & 1020(20) & $4338(6)$ & $520(100)$ \\
\hline$C(180)$ & $3907(15)$ & 411(14) & $4227(8)$ & $550(100)$ \\
\hline$C(181)$ & $3458(8)$ & $4795(12)$ & $2820(4)$ & $84(12)$ \\
\hline$C(182)$ & $3761(8)$ & $4735(12)$ & $2803(4)$ & $98(13)$ \\
\hline$C(183)$ & $3915(6)$ & $5022(12)$ & $2705(4)$ & $104(13)$ \\
\hline$C(184)$ & $3768(6)$ & $5370(10)$ & $2624(3)$ & $102(13)$ \\
\hline$C(185)$ & $3466(6)$ & $5431(11)$ & $2641(4)$ & $96(13)$ \\
\hline$C(186)$ & $3311(6)$ & $5143(13)$ & $2739(4)$ & $95(13)$ \\
\hline$C(187)$ & 3912(9) & $5692(13)$ & $2522(4)$ & $200(40)$ \\
\hline$C(188)$ & $4237(9)$ & $5550(20)$ & $2493(6)$ & $340(60)$ \\
\hline$C(189)$ & $3836(13)$ & $5647(19)$ & $2354(4)$ & $250(50)$ \\
\hline$C(190)$ & $3927(13)$ & $6211(12)$ & $2578(7)$ & $300(60)$ \\
\hline$C(191)$ & $1665(8)$ & $1625(10)$ & $4363(4)$ & $95(13)$ \\
\hline$C(192)$ & $1510(8)$ & $1595(9)$ & $4225(4)$ & $93(13)$ \\
\hline$C(193)$ & $1439(7)$ & $1160(11)$ & $4165(4)$ & $99(13)$ \\
\hline$C(194)$ & $1522(6)$ & $756(9)$ & $4241(4)$ & $94(13)$ \\
\hline$C(195)$ & $1676(8)$ & $786(10)$ & $4379(4)$ & 113(14) \\
\hline$C(196)$ & $1748(8)$ & $1220(12)$ & $4439(4)$ & $105(14)$ \\
\hline$C(197)$ & $1453(10)$ & $289(10)$ & $4188(5)$ & $290(50)$ \\
\hline$C(198)$ & $1324(14)$ & 292(16) & $4024(6)$ & $290(60)$ \\
\hline$C(199)$ & $1710(14)$ & $-13(12)$ & $4139(7)$ & $340(70)$ \\
\hline$C(200)$ & $1222(13)$ & $16(13)$ & $4285(7)$ & $370(70)$ \\
\hline
\end{tabular}




\begin{tabular}{|c|c|c|c|c|}
\hline$C(201)$ & 2919(7) & $2235(12)$ & $4568(3)$ & $74(11)$ \\
\hline$C(202)$ & $2767(5)$ & $2499(13)$ & $4673(4)$ & $79(11)$ \\
\hline$C(203)$ & 2902(7) & $2619(12)$ & $4811(3)$ & $84(12)$ \\
\hline$C(204)$ & $3189(7)$ & $2473(11)$ & $4843(3)$ & $85(12)$ \\
\hline$C(205)$ & $3341(6)$ & $2208(12)$ & $4737(4)$ & $90(12)$ \\
\hline$C(206)$ & $3206(7)$ & 2089(12) & $4600(4)$ & $83(12)$ \\
\hline$C(207)$ & $3349(11)$ & 2587(19) & $4982(4)$ & $520(90)$ \\
\hline$C(208)$ & $3146(15)$ & $2820(20)$ & $5101(4)$ & $560(100)$ \\
\hline$C(209)$ & $3420(14)$ & $2190(20)$ & $5090(4)$ & $560(100)$ \\
\hline$C(210)$ & $3614(12)$ & $2930(20)$ & $4963(6)$ & $570(100)$ \\
\hline$C(211)$ & $1580(9)$ & $3899(12)$ & $4726(3)$ & $83(12)$ \\
\hline$C(212)$ & $1306(9)$ & $3721(13)$ & $4766(4)$ & $110(14)$ \\
\hline$C(213)$ & $1183(7)$ & $3843(13)$ & 4904(4) & $116(14)$ \\
\hline$C(214)$ & $1333(8)$ & $4144(12)$ & $5002(3)$ & 113(14) \\
\hline$C(215)$ & $1607(8)$ & $4322(12)$ & $4963(4)$ & 101(13) \\
\hline$C(216)$ & $1730(7)$ & $4200(13)$ & $4825(4)$ & $93(12)$ \\
\hline$C(217)$ & $1220(11)$ & $4298(19)$ & $5147(4)$ & $410(70)$ \\
\hline$C(218)$ & $940(13)$ & $4020(30)$ & $5192(6)$ & $430(80)$ \\
\hline$C(219)$ & 1382(16) & $4130(30)$ & $5289(3)$ & $440(80)$ \\
\hline$C(220)$ & $1131(15)$ & $4830(20)$ & $5155(7)$ & $470(90)$ \\
\hline$C(221)$ & $4301(7)$ & $3108(14)$ & $4118(4)$ & 102(14) \\
\hline$C(222)$ & $4258(6)$ & $2654(15)$ & $4067(5)$ & $113(14)$ \\
\hline$C(223)$ & $4491(8)$ & $2406(12)$ & $4005(5)$ & $121(15)$ \\
\hline$C(224)$ & $4767(6)$ & 2612(13) & $3994(4)$ & $125(15)$ \\
\hline$C(225)$ & $4811(7)$ & $3066(13)$ & $4046(5)$ & $121(15)$ \\
\hline$C(226)$ & $4578(9)$ & $3314(12)$ & $4107(5)$ & $116(14)$ \\
\hline$C(227)$ & $5024(8)$ & 2378(19) & $3934(6)$ & $420(70)$ \\
\hline$C(228)$ & 4942(14) & 1910(20) & $3858(8)$ & $430(80)$ \\
\hline$C(229)$ & $5144(12)$ & $2560(30)$ & $3786(7)$ & $460(90)$ \\
\hline$C(230)$ & $5273(9)$ & $2260(20)$ & $4054(9)$ & $450(90)$ \\
\hline$C(231)$ & $3742(7)$ & $2957(18)$ & $4536(6)$ & 139(19) \\
\hline$C(232)$ & $3951(10)$ & $3142(15)$ & $4637(6)$ & $148(19)$ \\
\hline$C(233)$ & $4224(9)$ & $2926(16)$ & $4655(5)$ & 154(19) \\
\hline$C(234)$ & $4288(7)$ & $2526(14)$ & $4573(5)$ & $150(20)$ \\
\hline$C(235)$ & 4080(9) & $2342(15)$ & $4472(5)$ & 148(19) \\
\hline$C(236)$ & $3806(8)$ & 2557(19) & $4454(5)$ & 147(19) \\
\hline$C(237)$ & $4569(9)$ & $2280(20)$ & $4582(7)$ & $350(90)$ \\
\hline$C(238)$ & $4766(10)$ & $2470(30)$ & $4715(9)$ & $520(150)$ \\
\hline
\end{tabular}




\begin{tabular}{|c|c|c|c|c|}
\hline$C(239)$ & $4568(15)$ & $1800(20)$ & $4652(10)$ & $600(200)$ \\
\hline$C(240)$ & $4763(11)$ & $2320(30)$ & $4437(9)$ & $430(120)$ \\
\hline$C(241)$ & $630(6)$ & $3656(14)$ & $3324(4)$ & $87(12)$ \\
\hline$C(242)$ & $394(7)$ & $3912(11)$ & $3379(4)$ & $104(13)$ \\
\hline$C(243)$ & $123(6)$ & $3697(12)$ & $3402(4)$ & $102(13)$ \\
\hline$C(244)$ & $89(5)$ & $3225(12)$ & $3370(3)$ & $101(13)$ \\
\hline$C(245)$ & $325(7)$ & $2968(11)$ & $3315(4)$ & $94(12)$ \\
\hline$C(246)$ & $595(6)$ & $3184(14)$ & $3292(4)$ & $87(12)$ \\
\hline$C(247)$ & $-183(7)$ & $2969(17)$ & $3392(6)$ & $320(60)$ \\
\hline$C(248)$ & $-444(6)$ & $3300(20)$ & $3430(7)$ & $400(80)$ \\
\hline$C(249)$ & $-345(9)$ & $2790(20)$ & $3251(7)$ & $390(70)$ \\
\hline$C(250)$ & $-173(12)$ & $2600(20)$ & $3524(7)$ & $390(70)$ \\
\hline$C(251)$ & $3602(6)$ & 6393(11) & 2973(4) & $86(12)$ \\
\hline$C(252)$ & $3907(7)$ & $6337(10)$ & 2984(4) & $88(12)$ \\
\hline$C(253)$ & $4093(5)$ & $6679(10)$ & $2928(4)$ & 101(13) \\
\hline$C(254)$ & $3975(5)$ & $7076(8)$ & $2860(3)$ & 104(13) \\
\hline$C(255)$ & $3671(5)$ & $7132(10)$ & $2848(4)$ & $112(14)$ \\
\hline$C(256)$ & $3485(5)$ & $6791(12)$ & $2905(4)$ & $100(13)$ \\
\hline$C(257)$ & $4152(7)$ & $7454(10)$ & 2801(4) & $120(20)$ \\
\hline$C(258)$ & $4485(6)$ & $7325(14)$ & $2797(5)$ & $190(40)$ \\
\hline$C(259)$ & $4145(10)$ & $7526(14)$ & $2630(4)$ & $140(30)$ \\
\hline$C(260)$ & $4137(10)$ & $7924(10)$ & $2892(5)$ & $160(30)$ \\
\hline$C(261)$ & $2528(9)$ & $5396(18)$ & $4756(4)$ & 146(19) \\
\hline$C(262)$ & $2337(10)$ & $5125(15)$ & $4841(5)$ & 147(19) \\
\hline$C(263)$ & $2213(8)$ & $5307(14)$ & 4971(4) & 157(19) \\
\hline$C(264)$ & $2280(7)$ & $5759(14)$ & $5018(3)$ & $170(20)$ \\
\hline$C(265)$ & 2472(9) & $6029(14)$ & 4933(4) & $160(20)$ \\
\hline$C(266)$ & $2596(9)$ & $5848(18)$ & $4802(4)$ & 157(19) \\
\hline$C(267)$ & $2158(10)$ & $5980(18)$ & $5151(4)$ & $250(50)$ \\
\hline$C(268)$ & 1987(13) & $5630(20)$ & $5250(5)$ & $320(60)$ \\
\hline$C(269)$ & $2369(14)$ & $6080(20)$ & $5282(4)$ & $320(60)$ \\
\hline$C(270)$ & $1936(13)$ & $6390(20)$ & $5117(7)$ & $300(60)$ \\
\hline$C(271)$ & $1502(8)$ & 6006(13) & 2947(4) & $99(13)$ \\
\hline$C(272)$ & $1548(8)$ & $5855(10)$ & $2800(4)$ & $95(12)$ \\
\hline$C(273)$ & $1593(7)$ & $6178(11)$ & $2685(3)$ & $93(12)$ \\
\hline$C(274)$ & $1591(6)$ & $6653(10)$ & $2717(3)$ & 103(13) \\
\hline$C(275)$ & $1545(8)$ & $6804(11)$ & $2865(4)$ & 111(13) \\
\hline$C(276)$ & $1500(9)$ & $6480(14)$ & 2980(3) & 108(13) \\
\hline
\end{tabular}




\begin{tabular}{|c|c|c|c|c|}
\hline$C(277)$ & $1640(11)$ & $7015(14)$ & $2605(5)$ & $340(60)$ \\
\hline$C(278)$ & $1641(15)$ & $6820(20)$ & 2441(4) & $380(70)$ \\
\hline$C(279)$ & 1381(14) & $7329(17)$ & $2565(7)$ & $400(80)$ \\
\hline$C(280)$ & $1938(13)$ & $7288(17)$ & $2624(7)$ & $410(80)$ \\
\hline$C(281)$ & $3842(10)$ & $7108(10)$ & $3398(5)$ & $125(16)$ \\
\hline$C(282)$ & $3932(10)$ & $7432(13)$ & $3507(5)$ & $128(16)$ \\
\hline$C(283)$ & $3849(9)$ & $7897(12)$ & $3492(4)$ & $135(16)$ \\
\hline$C(284)$ & $3675(7)$ & $8039(10)$ & $3369(4)$ & $145(17)$ \\
\hline$C(285)$ & $3585(9)$ & $7715(12)$ & $3260(4)$ & $149(17)$ \\
\hline$C(286)$ & $3668(11)$ & $7250(11)$ & $3275(5)$ & $141(17)$ \\
\hline$C(287)$ & $3574(11)$ & $8514(11)$ & $3346(6)$ & $300(50)$ \\
\hline$C(288)$ & $3725(16)$ & $8859(11)$ & $3459(8)$ & $320(60)$ \\
\hline$C(289)$ & $3698(16)$ & $8777(14)$ & $3212(7)$ & $370(70)$ \\
\hline$C(290)$ & $3234(12)$ & $8590(18)$ & $3365(9)$ & $330(70)$ \\
\hline$C(291)$ & $3244(10)$ & $3246(11)$ & $2750(3)$ & $109(15)$ \\
\hline$C(292)$ & $3542(10)$ & $3336(13)$ & 2730(3) & $123(15)$ \\
\hline$C(293)$ & $3635(7)$ & $3570(11)$ & 2601(3) & $120(15)$ \\
\hline$C(294)$ & $3429(6)$ & $3716(8)$ & 2492(3) & $115(15)$ \\
\hline$C(295)$ & $3130(7)$ & $3626(11)$ & 2512(3) & $116(15)$ \\
\hline$C(296)$ & $3038(8)$ & $3392(13)$ & $2641(4)$ & $115(15)$ \\
\hline$C(297)$ & $3504(8)$ & $3969(11)$ & $2355(3)$ & $140(30)$ \\
\hline$C(298)$ & $3842(8)$ & $3984(16)$ & $2331(5)$ & $160(30)$ \\
\hline$C(299)$ & $3458(11)$ & $3718(16)$ & $2205(3)$ & $210(50)$ \\
\hline$C(300)$ & $3400(11)$ & 4491(11) & $2350(5)$ & $160(30)$ \\
\hline$C(301)$ & $3468(7)$ & $6470(12)$ & $4413(5)$ & $104(14)$ \\
\hline$C(302)$ & $3505(7)$ & $6550(13)$ & $4568(5)$ & $112(14)$ \\
\hline$C(303)$ & $3762(8)$ & $6759(12)$ & $4623(4)$ & $121(15)$ \\
\hline$C(304)$ & $3982(6)$ & 6889(9) & $4523(4)$ & $132(16)$ \\
\hline$C(305)$ & $3945(7)$ & $6809(13)$ & $4368(3)$ & $127(15)$ \\
\hline$C(306)$ & $3688(9)$ & $6600(14)$ & $4313(4)$ & $116(14)$ \\
\hline$C(307)$ & $4255(7)$ & $7116(14)$ & $4570(5)$ & $180(40)$ \\
\hline$C(308)$ & $4295(11)$ & $7120(20)$ & $4745(5)$ & $250(60)$ \\
\hline$C(309)$ & $4538(6)$ & $6840(20)$ & $4553(7)$ & $250(60)$ \\
\hline$C(310)$ & $4285(11)$ & $7638(14)$ & $4519(7)$ & $210(50)$ \\
\hline$C(311)$ & $1384(8)$ & 1972(10) & $3808(3)$ & $79(11)$ \\
\hline$C(312)$ & $1079(8)$ & 1976(9) & $3819(4)$ & $95(12)$ \\
\hline$C(313)$ & $911(6)$ & $1621(10)$ & $3753(3)$ & $95(13)$ \\
\hline$C(314)$ & $1050(6)$ & $1263(8)$ & $3675(3)$ & $99(13)$ \\
\hline
\end{tabular}




\begin{tabular}{|c|c|c|c|c|}
\hline$C(315)$ & $1355(6)$ & $1259(10)$ & $3663(4)$ & $100(13)$ \\
\hline$C(316)$ & $1523(6)$ & $1614(12)$ & $3730(4)$ & $87(12)$ \\
\hline$C(317)$ & 894(8) & $875(10)$ & $3604(4)$ & $120(20)$ \\
\hline$C(318)$ & $555(7)$ & $951(15)$ & $3602(6)$ & $180(40)$ \\
\hline$C(319)$ & $896(11)$ & $849(16)$ & $3432(4)$ & $300(70)$ \\
\hline$C(320)$ & $945(11)$ & 389(9) & $3683(6)$ & $210(50)$ \\
\hline$C(321)$ & $3533(9)$ & 4941(15) & $4721(3)$ & $108(14)$ \\
\hline$C(322)$ & $3494(10)$ & $5421(15)$ & $4730(3)$ & $120(15)$ \\
\hline$C(323)$ & $3447(10)$ & $5629(11)$ & $4871(4)$ & $134(15)$ \\
\hline$C(324)$ & $3440(7)$ & $5357(12)$ & $5001(3)$ & $134(16)$ \\
\hline$C(325)$ & $3480(9)$ & $4877(12)$ & 4992(4) & $128(15)$ \\
\hline$C(326)$ & $3526(10)$ & $4669(11)$ & $4852(4)$ & $117(14)$ \\
\hline$C(327)$ & $3399(12)$ & $5544(17)$ & $5152(4)$ & $300(70)$ \\
\hline$C(328)$ & $3309(16)$ & $6067(18)$ & $5148(6)$ & $530(160)$ \\
\hline C(329) & $3113(14)$ & $5420(20)$ & $5231(5)$ & $330(80)$ \\
\hline$C(330)$ & $3676(15)$ & $5520(20)$ & $5261(4)$ & $310(80)$ \\
\hline$C(331)$ & $724(8)$ & $5749(13)$ & $3274(4)$ & $94(13)$ \\
\hline$C(332)$ & $796(7)$ & $6214(13)$ & $3251(3)$ & $86(12)$ \\
\hline C(333) & $727(7)$ & $6427(10)$ & $3113(4)$ & 101(13) \\
\hline$C(334)$ & $585(6)$ & $6174(11)$ & 2998(3) & $110(14)$ \\
\hline$C(335)$ & $512(8)$ & $5708(11)$ & $3022(4)$ & $126(15)$ \\
\hline$C(336)$ & $582(9)$ & $5496(11)$ & $3160(5)$ & $114(14)$ \\
\hline C(337) & $499(10)$ & $6368(17)$ & $2852(4)$ & $300(50)$ \\
\hline C(338) & $632(14)$ & 6861(18) & $2828(6)$ & $360(70)$ \\
\hline C(339) & $659(14)$ & $6180(20)$ & $2714(3)$ & $290(60)$ \\
\hline$C(340)$ & $158(11)$ & $6420(20)$ & $2824(6)$ & $330(60)$ \\
\hline$C(341)$ & $1478(11)$ & $5570(20)$ & $4756(5)$ & $150(20)$ \\
\hline$C(342)$ & $1519(11)$ & $6050(20)$ & $4775(6)$ & $170(20)$ \\
\hline C(343) & $1332(11)$ & $6297(16)$ & $4869(6)$ & $190(20)$ \\
\hline$C(344)$ & $1105(9)$ & $6070(17)$ & 4943(4) & $190(20)$ \\
\hline$C(345)$ & $1064(11)$ & $5592(17)$ & $4923(6)$ & $190(20)$ \\
\hline$C(346)$ & $1251(13)$ & $5343(15)$ & $4830(6)$ & $170(20)$ \\
\hline$C(347)$ & $903(13)$ & $6300(20)$ & $5045(7)$ & $560(90)$ \\
\hline$C(348)$ & $940(20)$ & $6840(20)$ & $5039(10)$ & $590(110)$ \\
\hline$C(349)$ & $582(12)$ & $6340(30)$ & 4993(10) & $540(110)$ \\
\hline$C(350)$ & $939(19)$ & $6160(30)$ & $5217(6)$ & $590(110)$ \\
\hline$C(351)$ & $1255(6)$ & $2406(12)$ & $3301(3)$ & $67(10)$ \\
\hline$C(352)$ & $1005(7)$ & $2155(13)$ & $3340(3)$ & $75(11)$ \\
\hline
\end{tabular}




\begin{tabular}{|c|c|c|c|c|}
\hline$C(353)$ & $816(6)$ & 1983(12) & $3227(4)$ & $84(12)$ \\
\hline$C(354)$ & $878(6)$ & $2063(13)$ & $3075(3)$ & $76(11)$ \\
\hline$C(355)$ & $1128(7)$ & 2314(13) & $3036(3)$ & $79(11)$ \\
\hline$C(356)$ & $1316(6)$ & $2486(12)$ & $3149(3)$ & $73(11)$ \\
\hline$C(357)$ & $710(9)$ & $1812(15)$ & 2951(4) & $89(17)$ \\
\hline$C(358)$ & $393(11)$ & 2017(19) & $2955(7)$ & $130(30)$ \\
\hline$C(359)$ & $747(12)$ & 1983(18) & $2789(5)$ & $120(20)$ \\
\hline$C(360)$ & 671(14) & $1269(14)$ & 2962(7) & $150(30)$ \\
\hline$C(361)$ & $3186(12)$ & $6873(14)$ & $3899(7)$ & $160(20)$ \\
\hline$C(362)$ & $3425(14)$ & $7041(18)$ & $3818(6)$ & $180(20)$ \\
\hline$C(363)$ & $3598(11)$ & 7398(18) & $3879(6)$ & $190(20)$ \\
\hline$C(364)$ & $3532(10)$ & $7588(15)$ & $4019(6)$ & $190(20)$ \\
\hline$C(365)$ & $3292(12)$ & $7420(18)$ & $4100(5)$ & $180(20)$ \\
\hline$C(366)$ & $3119(11)$ & $7063(17)$ & $4039(7)$ & $160(20)$ \\
\hline$C(367)$ & $3702(15)$ & $7960(20)$ & 4093(9) & $590(100)$ \\
\hline$C(368)$ & $3932(16)$ & $8170(30)$ & $3984(12)$ & $570(110)$ \\
\hline$C(369)$ & $3550(20)$ & $8426(19)$ & $4110(11)$ & $610(110)$ \\
\hline$C(370)$ & $3880(20)$ & $7800(30)$ & $4238(10)$ & $630(110)$ \\
\hline$C(371)$ & $706(6)$ & $6328(12)$ & $3763(4)$ & $91(13)$ \\
\hline$C(372)$ & $562(7)$ & $5990(13)$ & $3847(3)$ & $89(13)$ \\
\hline$C(373)$ & 299(7) & $5797(11)$ & $3793(4)$ & $96(13)$ \\
\hline$C(374)$ & $180(6)$ & $5943(11)$ & $3655(4)$ & $109(14)$ \\
\hline$C(375)$ & $323(7)$ & $6281(12)$ & $3571(3)$ & $111(14)$ \\
\hline$C(376)$ & $586(7)$ & $6474(12)$ & $3624(4)$ & $94(13)$ \\
\hline$C(377)$ & $-91(8)$ & $5756(16)$ & $3589(5)$ & $280(50)$ \\
\hline$C(378)$ & $-257(9)$ & $5440(19)$ & $3702(7)$ & $340(70)$ \\
\hline$C(379)$ & $-353(7)$ & $6080(20)$ & $3577(7)$ & $320(60)$ \\
\hline$C(380)$ & $-50(12)$ & $5450(20)$ & $3442(6)$ & $330(70)$ \\
\hline$C(381)$ & $1149(6)$ & $4015(13)$ & $2900(4)$ & $70(11)$ \\
\hline$C(382)$ & $1056(7)$ & $3557(12)$ & 2920(4) & $90(12)$ \\
\hline$C(383)$ & $764(8)$ & $3437(10)$ & $2888(4)$ & $94(12)$ \\
\hline$C(384)$ & $566(6)$ & $3775(12)$ & 2836(4) & 107(13) \\
\hline$C(385)$ & $660(7)$ & $4233(11)$ & 2817(4) & 106(13) \\
\hline$C(386)$ & $951(8)$ & $4353(11)$ & $2849(4)$ & $94(12)$ \\
\hline$C(387)$ & $259(7)$ & $3681(19)$ & $2798(6)$ & $440(70)$ \\
\hline$C(388)$ & $165(10)$ & $3180(20)$ & $2847(8)$ & $480(90)$ \\
\hline$C(389)$ & $28(6)$ & $3900(20)$ & $2899(8)$ & $470(90)$ \\
\hline$C(390)$ & $177(11)$ & $3720(30)$ & $2625(7)$ & $490(90)$ \\
\hline
\end{tabular}




\begin{tabular}{|c|c|c|c|c|}
\hline$C(391)$ & $4250(8)$ & $5091(15)$ & $4376(4)$ & $103(14)$ \\
\hline$C(392)$ & $4407(9)$ & $4765(13)$ & $4461(5)$ & $122(15)$ \\
\hline C(393) & $4483(9)$ & $4859(13)$ & 4611(5) & 139(16) \\
\hline$C(394)$ & $4402(8)$ & $5281(14)$ & $4677(4)$ & $137(16)$ \\
\hline$C(395)$ & $4245(8)$ & $5608(12)$ & 4591(5) & $129(16)$ \\
\hline$C(396)$ & $4169(8)$ & $5513(14)$ & $4441(5)$ & $103(14)$ \\
\hline$C(397)$ & $4467(13)$ & $5410(20)$ & $4833(4)$ & $430(70)$ \\
\hline C(398) & $4683(16)$ & $5060(30)$ & 4911(5) & $470(90)$ \\
\hline$C(399)$ & $4684(15)$ & $5800(30)$ & $4860(6)$ & $450(90)$ \\
\hline$C(400)$ & 4192(16) & $5430(30)$ & 4939(4) & $490(90)$ \\
\hline $\mathrm{C}(401)$ & $3369(10)$ & 1992(12) & $3463(5)$ & $115(15)$ \\
\hline$C(402)$ & $3172(8)$ & $1721(15)$ & $3545(5)$ & $118(15)$ \\
\hline$C(403)$ & $3244(8)$ & $1264(14)$ & $3583(5)$ & $128(15)$ \\
\hline$C(404)$ & $3513(8)$ & $1078(12)$ & $3540(4)$ & $134(16)$ \\
\hline$C(405)$ & $3710(8)$ & $1348(14)$ & $3458(5)$ & $139(16)$ \\
\hline$C(406)$ & $3638(9)$ & $1805(14)$ & $3420(5)$ & $124(15)$ \\
\hline$C(407)$ & $3609(13)$ & $608(13)$ & $3577(7)$ & $280(70)$ \\
\hline$C(408)$ & $3357(17)$ & $313(15)$ & $3645(9)$ & $460(130)$ \\
\hline$C(409)$ & $3637(17)$ & $267(13)$ & $3446(9)$ & $510(150)$ \\
\hline$C(410)$ & $3872(16)$ & $570(20)$ & $3694(8)$ & $550(170)$ \\
\hline $\mathrm{C}(411)$ & $941(8)$ & $2577(11)$ & $4366(5)$ & $113(15)$ \\
\hline$C(412)$ & $1010(9)$ & $2548(12)$ & $4520(5)$ & $132(16)$ \\
\hline$C(413)$ & $943(9)$ & $2146(13)$ & $4600(4)$ & $143(17)$ \\
\hline$C(414)$ & $807(6)$ & 1772(10) & $4527(4)$ & $140(17)$ \\
\hline$C(415)$ & $737(8)$ & 1801(12) & $4373(4)$ & $138(17)$ \\
\hline$C(416)$ & $804(9)$ & $2203(14)$ & $4292(4)$ & $121(16)$ \\
\hline$C(417)$ & $733(10)$ & $1336(12)$ & 4601(5) & $220(50)$ \\
\hline$C(418)$ & $775(14)$ & $1368(19)$ & $4776(5)$ & $300(70)$ \\
\hline$C(419)$ & $408(11)$ & $1226(16)$ & $4618(6)$ & $180(40)$ \\
\hline$C(420)$ & $920(14)$ & $903(12)$ & $4551(8)$ & $380(100)$ \\
\hline $\mathrm{C}(421)$ & $832(8)$ & $5964(14)$ & $4402(5)$ & $124(16)$ \\
\hline$C(422)$ & $846(7)$ & $5781(16)$ & $4548(5)$ & $136(16)$ \\
\hline$C(423)$ & $598(7)$ & 5574(14) & 4611(4) & 138(16) \\
\hline$C(424)$ & $336(6)$ & $5551(10)$ & $4527(3)$ & $132(16)$ \\
\hline$C(425)$ & $322(8)$ & $5735(14)$ & 4381(3) & $134(16)$ \\
\hline$C(426)$ & $570(9)$ & $5941(16)$ & $4318(4)$ & $137(16)$ \\
\hline$C(427)$ & $68(7)$ & $5336(14)$ & $4581(5)$ & $160(30)$ \\
\hline$C(428)$ & $93(11)$ & $5210(20)$ & $4753(5)$ & $270(70)$ \\
\hline
\end{tabular}




$\begin{array}{lccll}\mathrm{C}(429) & -192(7) & 5660(20) & 4611(7) & 220(50) \\ \mathrm{C}(430) & -24(11) & 4871(17) & 4499(7) & 270(60) \\ \mathrm{C}(431) & 2139(13) & 6782(14) & 4341(6) & 158(19) \\ \mathrm{C}(432) & 2330(10) & 7116(19) & 4284(7) & 173(19) \\ \mathrm{C}(433) & 2236(10) & 7576(17) & 4268(6) & 180(19) \\ \mathrm{C}(434) & 1952(10) & 7701(13) & 4309(5) & 180(20) \\ \mathrm{C}(435) & 1761(9) & 7366(17) & 4367(6) & 173(19) \\ \mathrm{C}(436) & 1855(12) & 6906(15) & 4383(6) & 159(18) \\ \mathrm{C}(437) & 1836(15) & 8172(15) & 4299(8) & 520(80) \\ \mathrm{C}(438) & 2050(20) & 8500(16) & 4211(11) & 520(100) \\ \mathrm{C}(439) & 1591(19) & 8260(20) & 4183(10) & 520(100) \\ \mathrm{C}(440) & 1780(20) & 8418(19) & 4455(10) & 510(100)\end{array}$

